\title{
Predictores de recuperación subjetiva en la esquizofrenia
}

\author{
Olimpia Díaz-Mandado ${ }^{1}$, Marta Nieto-Moreno ${ }^{1}$, Ignacio Montorio ${ }^{2}$ y Jose A. Periáñez ${ }^{3}$ \\ ${ }^{1}$ Fundación Sociosanitaria de Castilla la Mancha,Toledo, España \\ ${ }^{2}$ Universidad Autónoma de Madrid, Madrid, España \\ ${ }^{3}$ Universidad Complutense de Madrid, Madrid, España
}

Resumen: El objetivo del presente trabajo fue analizar los factores que promueven la experiencia subjetiva de recuperación en personas con diagnóstico de esquizofrenia, y su relación con los indicadores de recuperación objetiva. Para ello, tras revisar la literatura existente, se evalúo el valor predictivo de diferentes variables psicosociales y clínicas sobre la recuperación subjetiva en una muestra de 43 personas con esquizofrenia. El análisis de regresión múltiple reveló que el uso de estrategias adaptativas de afrontamiento, un bajo estigma internalizado y una elevada autoestima predijeron un $55 \%$ de la variable recuperación subjetiva $(F=12,5 ; p<0,0001)$. Los síntomas negativos y el funcionamiento social no predijeron recuperación subjetiva, pese a la presencia de correlaciones significativas. Se discuten los resultados en relación con la superior predicción de los indicadores subjetivos de recuperación, y se reflexiona sobre las implicaciones de estos resultados en el tratamiento y la promoción de mejores expectativas de recuperación en esta población.

Palabras clave: Afrontamiento; esquizofrenia; estigma internalizado; ISMI; RAS; recuperación subjetiva.

\section{Predictors of subjective recovery in schizophrenia}

\begin{abstract}
The aim of the present study was to analyze factors that promote the experience of subjective recovery in people with a diagnosis of schizophrenia, and their relationship with objective recovery indicators. To do so, and after reviewing the existing literature, the predictive value of a set of different clinical and psychosocial variables on subjective recovery was evaluated of a sample of 43 adults with schizophrenia. Multiple regression analysis revealed that using adaptive coping strategies, low internalized stigma, and high self-esteem predicted $55 \%$ of the variance of subjective recovery $(F=12.5 ; p<0.0001)$. Negative symptoms and social functioning did not predict subjective recovery, in spite of significant correlations. Both the lack of predictive value of objective compared to subjective recovery measures, and the implications of the present findings for the treatment and promotion of increased recovery expectancies in this population are discussed.
\end{abstract}

Keywords: Coping; internalized stigma; ISMI; RAS; schizophrenia; subjective recovery.

\section{Introducción}

El concepto de recuperación en esquizofrenia ha sido tradicionalmente definido en términos de remisión de síntomas y consecución de resultados psicosociales ( $\mathrm{Li}$ berman, Kopelowicz, Ventura y Gutkind, 2002). Esta de- finición comúnmente utilizada en los ámbitos aplicados y de investigación refleja la recuperación como un resultado en función de si se cumplen ciertos criterios objetivos. Sin embargo diferentes autores han propuesto otras definiciones complementarias basadas en la perspectiva de las personas con esquizofrenia y sus familiares que enfa-
Recibido: 13 julio 2014; aceptado: 11 marzo 2015.

Correspondencia: Olimpia Díaz-Mandado, Centro de Rehabilitación Psicosocial y Laboral de Toledo, Fundación Sociosanitaria de Castilla la Mancha, Avda. Purísima Concepción 31, 45006 Toledo. Correo-e: olimpia.diaz@hotmail.com
Agradecimientos: Los autores agradecen a los usuarios de los Centros de Rehabilitación Psicosocial y Laboral (CRPSL) de la provincia de Toledo su participación en este estudio, a la Fundación Sociosanitaria de Castilla la Mancha su apoyo técnico y logístico, y a los psicólogos Eva M. Cabezuelo, Sandra Díaz, Manuel Hernández, Marga Hernández, Enrique Mellado, Marta Nicolás y Sofía Pérez su participación en la recogida de datos. 
tizan, más allá de la consecución de ciertos resultados, el proceso continuo de cambio en la identidad personal (Silverstein y Bellack, 2008). Este concepto denominado recuperación psicológica (Andresen, Oades y Caputi, 2003) o subjetiva (Roe, Mashiach-Eizenberg y Lysaker, 2011) hace referencia a la capacidad percibida para hacer frente al trastorno y sus consecuencias, el derecho de la persona a construir por sí misma una vida con sentido, con esperanza y optimismo sobre el futuro, la capacidad para asumir responsabilidad y la confianza para llevar una vida satisfactoria, todo ello independientemente de la gravedad del trastorno (SAMHSA, 2005)

Bajo este nuevo marco conceptual de la recuperación, diferentes autores reconocen la necesidad de ampliar las medidas clínicas objetivas con nuevos instrumentos que incluyan las percepciones y expectativas de las personas con esquizofrenia sobre su propia recuperación (Andresen, Caputi y Oades, 2010). Sin embargo hasta la fecha son pocas las herramientas existentes dirigidas a evaluar la experiencia subjetiva de recuperación. En este sentido uno de los instrumentos que más atención ha acaparado entre los investigadores de este ámbito ha sido la Escala de Evaluación de la Recuperación (Recovery Assessment Scale, RAS; Corrigan, Giffort,
Rashid, Leary y Okeke, 1999). Esta escala permite evaluar las percepciones y expectativas sobre el proceso de recuperación considerando de forma específica constructos como la esperanza, la confianza en uno mismo y en los demás, la disposición a pedir ayuda, la orientación hacia la consecución de metas personales, así como la percepción de no sentirse dominado por los síntomas del trastorno mental. En este sentido el RAS proporciona una medida adecuada para evaluar la experiencia subjetiva de recuperación, presentando unas características psicométricas y conceptuales sólidas (Chiba, Miyamoto y Kawakami, 2010; Corrigan et al., 1999; Corrigan, Salzer, Ralph, Sangster y Keck, 2004; McNaught, Caputi, Oades y Deane, 2007) y mostrando validez convergente con otras medidas de recuperación subjetiva existentes (Andresen et al., 2010; McNaught et al., 2007) como por ejemplo el Mental Health Recovery Measure (MHRM; Young y Bullock, 2003) o el Self-identified Stage of Recovery (SISR; Andresen, Caputi y Oades, 2006).

En el momento actual existe un interés creciente por establecer qué variables determinan la recuperación subjetiva en las personas con esquizofrenia dada su importancia en el establecimiento de modelos teóricos de recuperación (Silverstein y Bellack, 2008) y en la mejora

Tabla 1. Revisión de investigaciones que han estudiado la relación del RAS con otras variables

\begin{tabular}{|c|c|c|c|c|}
\hline $\begin{array}{l}\text { Autores } \\
\text { (año) }\end{array}$ & $\begin{array}{l}\text { Muestra }(\mathrm{N}) \\
\text { Análisis }\end{array}$ & Medidas & Resultados & Conclusiones \\
\hline $\begin{array}{l}\text { (1) Corrigan } \\
\text { et al., (1999) }\end{array}$ & $\begin{array}{l}\text { TMG }(n=35) \\
\text { Regr. }\end{array}$ & $\begin{array}{l}\text { RAS-41 } \\
\text { ES, RSES, QOLI, } \\
\text { SSQ, BPRS, } \\
\text { NARA, } \\
\text { Vocabulary } \\
\text { Subtest WAIS-R, } \\
\text { GAF }\end{array}$ & $\begin{array}{l}\text { Correlación entre RAS y ES- Self- } \\
\text { orientation }(r=-0.71) \text {, RSES }(r=0.55) \text {, } \\
\text { SSQ-Size of Support Network } \\
(r=0.48) \text {, BPRS }(r=-0.44) \text {, QOLI } \\
(r=0.62) \text { y edad }(r=0.34) \text {. } \\
\text { RAS predicho por RSES }(r=0.45), \\
\text { ES- Self-orientation }(r=0.25) \text { y edad }(r \\
=0.08)\end{array}$ & $\begin{array}{l}\text { Relación entre RAS y 'autoestima', } \\
\text { 'empoderamiento', 'tamaño de la red } \\
\text { social', 'calidad de vida', 'síntomas } \\
\text { psiquiátricos'y 'edad' } \\
\text { RAS fue predicho por 'autoestima', } \\
\text { 'empoderamiento 'y 'edad'. }\end{array}$ \\
\hline $\begin{array}{l}\text { (2) Ritsher } \\
\text { et al., (2003) }\end{array}$ & $\begin{array}{l}\text { TMG } \\
(n=127) \\
\text { Corr. }\end{array}$ & $\begin{array}{l}\text { RAS-41 } \\
\text { ISMI, CES-D, } \\
\text { RSES, PDD, BU }\end{array}$ & $\begin{array}{l}\text { Correlación entre ISMI y RAS } \\
(r=-0.49)\end{array}$ & $\begin{array}{l}\text { Relación entre RAS y 'estigma } \\
\text { internalizado'. }\end{array}$ \\
\hline $\begin{array}{l}\text { (3) Corrigan } \\
\text { et al., }(2004)\end{array}$ & $\begin{array}{l}\text { TMG } \\
(n=1824) \\
\text { Regr. }\end{array}$ & $\begin{array}{l}\text { RAS-41 } \\
\text { (Punt. } 5 \text { factores) } \\
\text { HHI, ES, QOLI, } \\
\text { MLS, Hopkins } \\
\text { Symptom } \\
\text { Checklist }\end{array}$ & $\begin{array}{l}\text { RAS-Esperanza predicho por HHI } \\
(\beta=0.41), \text { MLS }(\beta=0.18), E S(\beta=0.18) \text {, } \\
\text { Hopkins Symptom Checklist }(\beta=-0.15) \\
\text { y QOLI }(\beta=0.06) \text {. } \\
\text { RAS-Ayuda predicho por HHI } \\
\text { ( } \beta=0.37) \text { and QOLI }(\beta=0.11) \\
\text { RAS-Metas predicho por HHI } \\
\text { ( } \beta=0.36), \text { MLS }(\beta=0.26) \text { y ES }(\beta=0.16) \text {. } \\
\text { RAS-Otros predicho por HHI }(\beta=0.42) \text {, } \\
\text { QOLI }(\beta=0.20) \text { y ES }(\beta=0.11) \\
\text { RAS-No dominación predicho por } \\
\text { HHI }(\beta=0.26), \text { Hopkins Symptom } \\
\text { Checklist }(\beta=-0.20), \operatorname{MLS}(\beta=0.10) \text {, } \\
\text { QOLI }(\beta=0.09) \text { y ES }(\beta=0.06) \text {. }\end{array}$ & $\begin{array}{l}\text { Todos los factores del RAS fueron } \\
\text { predichos por 'esperanza'. } \\
\text { Dos factores del RAS (Esperanza y No } \\
\text { dominación) fueron predichos por } \\
\text { 'síntomas psiquiátricos'. }\end{array}$ \\
\hline
\end{tabular}


Tabla 1. Revisión de investigaciones que han estudiado la relación del RAS con otras variables

\begin{tabular}{|c|c|c|c|c|}
\hline $\begin{array}{l}\text { Autores } \\
\text { (año) }\end{array}$ & $\begin{array}{c}\text { Muestra (N) } \\
\text { Análisis }\end{array}$ & Medidas & Resultados & Conclusiones \\
\hline $\begin{array}{l}(4) \text { Corrigan } \\
\text { y Phelan } \\
(2004)\end{array}$ & $\begin{array}{l}\text { TMG } \\
(n=176) \\
\text { Regr. }\end{array}$ & $\begin{array}{l}\text { RAS-41 } \\
\text { (Punt. } 5 \text { factores) } \\
\text { BPRS, SNS }\end{array}$ & $\begin{array}{l}\text { Correlación entre RAS-Esperanza, } \\
\text { RAS-Ayuda, RAS-Metas y RAS-Otros } \\
\text { con SNS-Tamaño de la red social } \\
\text { general ( } r>0.14) \text {. } \\
\text { Correlación entre todos los factores del } \\
\text { RAS con SNS-Percepción de } \\
\text { satisfacción con la red social }(r>0.28)\end{array}$ & $\begin{array}{l}\text { Relación entre factores del RAS y } \\
\text { 'tamaño de la red social general' y con } \\
\text { 'percepción de satisfacción con la red } \\
\text { social' }\end{array}$ \\
\hline $\begin{array}{l}\text { (5) Corrigan } \\
(2006)\end{array}$ & $\begin{array}{l}\text { TMG } \\
(n=1824) \\
\text { Regr. }\end{array}$ & $\begin{array}{l}\text { RAS-41 } \\
\text { (Punt. } 5 \text { factores) } \\
\text { ES, PPS, Variables } \\
\text { sociodemográficas }\end{array}$ & $\begin{array}{l}\text { RAS-Esperanza fue predicho } \\
\left(R^{2}=0,04\right) \text { por PPS }(B=0,088) \text {, género } \\
(B=0,087) \text { e raza }(B=-0,12) \text {. } \\
\text { RAS-Ayuda predicho }\left(R^{2}=0,01\right) \text { por } \\
\text { PPS }(B=0,073) \text { y raza }(B=-0,074) \text {. } \\
\text { RAS-Metas predicho }\left(R^{2}=0,04\right) \text { por } \\
\text { PPS }(B=0,12) \text {, raza }(B=-0,087) \text { y edad } \\
(B=-0,053) \text {. } \\
\text { RAS-Otros predicho }\left(R^{2}=0,03\right) \text { por } \\
\text { PPS }(B=0,14) \text {. }\end{array}$ & $\begin{array}{l}\text { Variables sociodemográficas y la } \\
\text { participación en 'ayuda mutua' } \\
\text { explicaron poca varianza del RAS. }\end{array}$ \\
\hline $\begin{array}{l}\text { (6) } \\
\text { McNaught } \\
\text { et al., (2007) }\end{array}$ & $\begin{array}{l}\text { TMG } \\
(n=168) \\
\text { Corr. }\end{array}$ & $\begin{array}{l}\text { RAS- } 24 \\
\text { (Punt. } 5 \text { factores) } \\
\text { K-10, MHRM, } \\
\text { SRM, HoNOS }\end{array}$ & $\begin{array}{l}\text { RAS-SRM: Correlación entre } \\
\text { RAS-Esperanza y -Metas con todas las } \\
\text { subescalas de SRM }(r>0.21) \text {. } \\
\text { Correlación entre RAS-Otros con } \\
\text { SRM-Esperanza, -Identidad y } \\
\text {-Responsabilidad ( } r>0.17) \text {. } \\
\text { Correlación entre RAS-Ayuda y } \\
\text { SRM-Identidad y -Responsabilidad } \\
(r>0.23) \text {. } \\
\text { RAS-MHRM: Correlación entre todos } \\
\text { los factores del RAS con los del } \\
\text { MHRM ( } r>0.20) \text {. } \\
\text { Correlación entre RAS-Esperanza y } \\
\text { HoNOS-Síntomas y -Conducta } \\
(r>-0.22) \text {. } \\
\text { Correlación entre RAS-No dominación } \\
\text { y HoNOS-Síntomas }(r=-0.19) \text {. } \\
\text { Correlación entre RAS-Esperanza, } \\
\text { RAS-Metas y RAS-No dominación } \\
\text { con K-10 ( } r=-0.43, r=-0.26, r=-0.21) \text {. }\end{array}$ & $\begin{array}{l}\text { Relación entre RAS con dos medidas } \\
\text { de recuperación subjetiva. } \\
\text { Pequeña relación entre dos factores del } \\
\text { RAS con 'funcionamiento social' y } \\
\text { entre tres factores del RAS y síntomas } \\
\text { depresivos y ansiosos. } \\
\text {. }\end{array}$ \\
\hline $\begin{array}{l}\text { (7) Hendryx } \\
\text { et al., (2009) }\end{array}$ & $\begin{array}{l}\text { TMG } \\
(n=153) \\
\text { Regr. }\end{array}$ & $\begin{array}{l}\text { RAS-41 } \\
\text { SF-12, } \\
\text { Satisfacción con } \\
\text { los clínicos, } \\
\text { WQLQ, } \\
\text { Participación en } \\
\text { actividades, } \\
\text { Variables } \\
\text { sociodemográficas. }\end{array}$ & $\begin{array}{l}\text { Correlación entre RAS y Participación } \\
\text { en actividades }(r=0.47), \text { WQLQ- } \\
\text { Soporte social }(r=0.44), y \text {-Red social } \\
(r=0.36) \text {. } \\
\text { RAS predicho por SFS-12-Salud } \\
\text { Mental, WQLQ-Soporte social, } \\
\text { Participación en actividades y la } \\
\text { interacción entre Participación en } \\
\text { actividades y WQLQ-Soporte social } \\
\left(\mathrm{R}^{2}=0.43\right) .\end{array}$ & $\begin{array}{l}\text { RAS predicho por 'el componente de } \\
\text { salud mental de la calidad de vida', } \\
\text { 'soporte social' y 'participación en } \\
\text { actividades'. }\end{array}$ \\
\hline $\begin{array}{l}\text { (8) Chiba et } \\
\text { al., (2010) }\end{array}$ & $\begin{array}{l}\text { TMG } \\
(n=209) \\
\text { Corr. }\end{array}$ & $\begin{array}{l}\text { RAS-24 } \\
\text { HHI, ES, } \\
\text { Resilience Scale, } \\
\text { SF-8, BASIS-32 }\end{array}$ & $\begin{array}{l}\text { Correlación entre RAS y HHI }(r=0.89) \text {, } \\
\text { ES }(r=0.76) \text {, Resilience Scale }(r=0.76) \text {, } \\
\text { SF-8-Componente salud mental } \\
(r=0.45) \text { y BASIS-32 }(r=-0.58) .\end{array}$ & $\begin{array}{l}\text { Relación entre RAS y 'esperanza', } \\
\text { 'empoderamiento', 'resiliencia', 'el } \\
\text { componente de salud mental de la } \\
\text { calidad de vida', 'síntomas } \\
\text { psiquiátricos' y deterioro funcional'. }\end{array}$ \\
\hline $\begin{array}{l}\text { (9) Lloyd et } \\
\text { al., (2009) }\end{array}$ & $\begin{array}{l}\text { TMG } \\
(n=161) \\
\text { Corr. } \\
\text { ANOVA }\end{array}$ & $\begin{array}{l}\text { RAS-24 } \\
\text { (Punt. } 5 \text { factores) } \\
\text { CIM, ES, } \\
\text { CANSAS, Edad, } \\
\text { género, situación } \\
\text { laboral y } \\
\text { diagnóstico. }\end{array}$ & $\begin{array}{l}\text { Correlación entre RAS y ES }(r=0.58) \text {, } \\
\text { CIM }(r=0.38) \text { y CANSAS }(r=-0.51) \\
\text { Diferencias en el RAS entre grupos } \\
\text { según la situación laboral y }(\mathrm{F}=7.38) \text { y } \\
\text { diagnóstico }(\mathrm{F}=16.5) \text {. }\end{array}$ & $\begin{array}{l}\text { Relación entre RAS y } \\
\text { 'empoderamiento', niveles de } \\
\text { 'participación en la comunidad' y } \\
\text { 'necesidades satisfechas'. } \\
\text { Relación entre RAS con situación } \\
\text { laboral y diagnóstico principal de } \\
\text { trastorno afectivo bipolar. }\end{array}$ \\
\hline
\end{tabular}


Tabla 1. Revisión de investigaciones que han estudiado la relación del RAS con otras variables

\begin{tabular}{|c|c|c|c|c|}
\hline $\begin{array}{l}\text { Autores } \\
\text { (año) }\end{array}$ & $\begin{array}{c}\text { Muestra (N) } \\
\text { Análisis }\end{array}$ & Medidas & Resultados & Conclusiones \\
\hline $\begin{array}{l}\text { (10) Muñoz } \\
\text { et al. (2010) }\end{array}$ & $\begin{array}{l}\text { TMG } \\
(n=108) \\
\text { Ecuaciones } \\
\text { Estructurales }\end{array}$ & $\begin{array}{l}\text { RAS-41 } \\
\text { AQ-27, ISMI, } \\
\text { SFS, BUS, EDD } \\
\text { Variables latentes: } \\
\text { Estigma social e } \\
\text { internalizado, } \\
\text { Discriminación, } \\
\text { Expectativas de } \\
\text { recuperación, } \\
\text { Empoderamiento, } \\
\text { Resultados } \\
\text { conductuales. }\end{array}$ & $\begin{array}{l}\text { Correlación entre RAS y AQ-27- } \\
\text { Evitación }(r=-0.26) \text {, Experiencias de } \\
\text { discriminación }(r=-0.20) \text {,SFS- } \\
\text { Funcionamiento social }(r=0.38) \text {, } \\
\text { SFS-Autonomía personal }(r=0.31) \text {, e } \\
\text { ISMI }(r=-0.51) \text {. } \\
\text { RAS e ISMI predicen los resultados de } \\
\text { SFS-Funcionamiento social y SFS- } \\
\text { Funcionamiento social (Efectos } \\
\text { directos del RAS=0.31) }\end{array}$ & $\begin{array}{l}\text { Relación entre RAS y 'estigma social', } \\
\text { 'experiencias de discriminación', } \\
\text { 'funcionamiento social' y 'estigma } \\
\text { internalizado'. } \\
\text { Las expectativas de recuperación y el } \\
\text { estigma internalizado predicen } \\
\text { directamente resultados conductuales } \\
\text { como el 'funcionamiento social' y la } \\
\text { 'autonomía personal'. }\end{array}$ \\
\hline $\begin{array}{l}(11) \\
\text { Andresen et } \\
\text { al., (2010) }\end{array}$ & $\begin{array}{l}\text { TMG } \\
(n=281) \\
\text { Corr. }\end{array}$ & $\begin{array}{l}\text { RAS-41 } \\
\text { (Punt 5. factores) } \\
\text { MHRM, SISR, } \\
\text { HoNOS, LSP-16, } \\
\text { GAF, K-10 }\end{array}$ & $\begin{array}{l}\text { Correlación entre RAS y MHRM } \\
(r=0.89), \text { SISR-B }(r=0.70), \text { SISR-A } \\
(r=0.40) \text { y K-10 }(r=0.33)\end{array}$ & $\begin{array}{l}\text { Relación entre RAS con dos medidas } \\
\text { de recuperación subjetiva. } \\
\text { Relación entre RAS y síntomas } \\
\text { depresivos y ansiosos. }\end{array}$ \\
\hline $\begin{array}{l}\text { (12) Roe et } \\
\text { al., (2011) }\end{array}$ & $\begin{array}{l}\text { ESQ }(n=159) \\
\text { Regr. }\end{array}$ & $\begin{array}{l}\text { RAS-20 } \\
\text { (Punt. } 4 \text { factores) } \\
\\
\text { BPRS-E, MSPSS, } \\
\text { GAF, S-SELAS, } \\
\text { MANSA, Edad, } \\
\text { Diagóstico, Edad } \\
\text { del diagnóstico. }\end{array}$ & $\begin{array}{l}\text { Correlación entre BPRS-Humor y RAS } \\
\text { total y RAS-Esperanza }(r=-0.17 \text {, } \\
r=-0.21) \text { y entre BPRS-Animación y } \\
\text { RAS-Ayuda }(r=0,18) \text {. } \\
\text { Correlación entre RAS y MSPSS } \\
(r=0.33) \text { y S-SELAS }(r=-0.32) \text {. } \\
\text { RAS fue predicho por la interacción } \\
\text { entre síntomas y edad de diagnóstico. } \\
(\beta=0.26, \mathrm{t}=2.67, \mathrm{p}<0.01) \text {. } \\
\text { Correlación entre MANSA y RAS } \\
\text { controlada MSPSS }(\beta=0.48) \text {. } \\
\text { Correlación entre MSPSS yAS } \\
\text { controlada MANSA ( } \beta=0.18) \text { pero } \\
\text { reducida desde } \beta=0.33 \text { a } \beta=0.18 \\
(\mathrm{Z}=3.44) \text {. } \\
\text { Correlación entre S-SELAS y RAS } \\
\text { disminuida desde } \beta=-0.32 \text { a } \beta=-0.12 \\
\text { cuando se controló MANSA } \\
(\mathrm{Z}=-4.26) \text {. }\end{array}$ & $\begin{array}{l}\text { Ausencia de relación directa entre la } \\
\text { puntuación total de la sintomatología } \\
\text { (BPRS) y el RAS, solo entre algunas } \\
\text { de sus factores. } \\
\text { Relación entre RAS y 'soporte social' } \\
\text { y 'soledad' } \\
\text { La edad del diagnóstico modera la } \\
\text { relación entre la 'severidad de los } \\
\text { síntomas' y RAS. } \\
\text { El impacto del 'soporte social' y la } \\
\text { 'soledad' sobre RAS fue mediada por } \\
\text { la 'calidad de vida'. }\end{array}$ \\
\hline $\begin{array}{l}\text { (13) Connell } \\
\text { et al., (2011) }\end{array}$ & $\begin{array}{l}\text { TMG } \\
(n=234) \\
\text { ANOVA }\end{array}$ & $\begin{array}{l}\text { RAS-41 } \\
\text { (Punt. } 5 \text { factores) } \\
\text { Situación laboral }\end{array}$ & $\begin{array}{l}\text { No diferencias entre trabajadores y no } \\
\text { trabajadores en RAS. } \\
\text { Diferencia en RAS-Otros y RAS- } \\
\text { Ayuda entre trabajadores y no } \\
\text { trabajadores }(F(1,229)=7.42),(F(1 . \\
231)=3.21) \text {. }\end{array}$ & $\begin{array}{l}\text { Los trabajadores tuvieron puntuaciones } \\
\text { más bajas que los no trabajadores en } \\
\text { RAS-Otros y RAS-Ayuda. }\end{array}$ \\
\hline $\begin{array}{l}\text { (14) Mukolo } \\
\text { et al., (2011) }\end{array}$ & $\begin{array}{l}\text { TMG } \\
(n=110) \\
\text { Regr. }\end{array}$ & $\begin{array}{l}\text { RAS- } 24 \\
\text { (Punt. } 4 \text { factores) } \\
\text { RSES, } \\
\text { Participación } \\
\text { actividades ayuda } \\
\text { mutua. }\end{array}$ & $\begin{array}{l}\text { Correlación entre RAS y RSES- } \\
\text { Global }(r=0.62) \text { y -Positiva }(r=0.88) \text {. } \\
\text { RSES-Positiva predicha por RAS- } \\
\text { Esperanza }(B=0.59) \text { y RAS-Ayuda } \\
(B=0.37)\left(\mathrm{R}^{2}=0.85\right) \text {. } \\
\text { RSES-Global predicha por RAS- } \\
\text { Esperanza }\left(B=0.57, \mathrm{R}^{2}=0.47\right)\end{array}$ & $\begin{array}{l}\text { Relación entre RAS y autoestima } \\
\text { positiva y global. } \\
\text { Dos factores del RAS predicen la } \\
\text { autoestima positiva. Un factor del RAS } \\
\text { predice la autoestima global. }\end{array}$ \\
\hline $\begin{array}{l}\text { (15) Cavelti } \\
\text { et al., (2012) }\end{array}$ & $\begin{array}{l}\text { ESQ }(n=142) \\
\text { Ecuaciones } \\
\text { estructurales }\end{array}$ & $\begin{array}{l}\text { RAS-24 } \\
\text { (Punt. } 3 \text { factores) } \\
\text { SUMD, CDSS, } \\
\text { BDI-II, SWN-K, } \\
\text { IPQS, PANSS } \\
\text { Variables latentes: } \\
\begin{array}{l}\text { Insight, Síntomas } \\
\text { depresivos y } \\
\text { psicóticos, Actitud } \\
\text { recuperación. }\end{array}\end{array}$ & $\begin{array}{l}\text { Síntomas depresivos predichos por } \\
\text { Insight }(\mathrm{B}=1.7) \text {, Síntomas psicóticos } \\
(\mathrm{B}=2) \text {, Actitud de recuperación } \\
(\mathrm{B}=-0.95) \text {, Actitud de recuperación } \times \\
\text { Insight }(\mathrm{B}=-0.16) \text { y Actitud de } \\
\text { recuperación } \times \text { Síntomas psicóticos } \\
(\mathrm{B}=-0.09) \text {. }\end{array}$ & $\begin{array}{l}\text { La asociación de altos niveles de } \\
\text { conciencia de enfermedad o de } \\
\text { síntomas psicóticos con altos niveles } \\
\text { de síntomas depresivos disminuye } \\
\text { cuando tres factores del RAS } \\
\text { (Esperanza, Metas y No dominación) } \\
\text { aumentan. }\end{array}$ \\
\hline
\end{tabular}


Tabla 1. Revisión de investigaciones que han estudiado la relación del RAS con otras variables

\begin{tabular}{|c|c|c|c|c|}
\hline $\begin{array}{l}\text { Autores } \\
\text { (año) }\end{array}$ & $\begin{array}{c}\text { Muestra (N) } \\
\text { Análisis }\end{array}$ & Medidas & Resultados & Conclusiones \\
\hline $\begin{array}{l}\text { (16) Roe et } \\
\text { al., (2012) }\end{array}$ & $\begin{array}{l}\text { TMG } \\
(n=158) \\
\text { Regr. }\end{array}$ & $\begin{array}{l}\text { RAS-20 } \\
\text { (Punt. } 4 \text { factores) } \\
\text { MSPS, BPRS, } \\
\text { MSAQL, SELS- } \\
\text { SV, GAF }\end{array}$ & $\begin{array}{l}\text { RAS-Esperanza predicha por MSAQL } \\
\left(\beta=0.46, \mathrm{R}^{2}=0.23\right) \text {. } \\
\text { RAS-Ayuda predicha por MSAQL y } \\
\text { MSPS }\left(\beta>0.24, \mathrm{R}^{2}=0.14\right) \text {. } \\
\text { RAS-Otros predicha por SELS-SV y } \\
\text { MSPS }\left(\beta=-0.23 ; \beta=0.20 ; \mathrm{R}^{2}=0.21\right) \\
\text { RAS-No dominación predicha por } \\
\text { MSAQL }\left(\beta=0.58, \mathrm{R}^{2}=0.28\right) \text {. }\end{array}$ & $\begin{array}{l}\text { RAS-Esperanza, RAS-Ayuda y } \\
\text { RAS-No dominación predicha por } \\
\text { 'calidad de vida'. } \\
\text { RAS-Otros predicha por 'soledad } \\
\text { emocional y social' y 'soporte social'. }\end{array}$ \\
\hline $\begin{array}{l}\text { (17) Kukla } \\
\text { et al., (2013) }\end{array}$ & $\begin{array}{l}\text { ESQ } \\
(n=46) \\
\text { Corr. } \\
\text { ANOVA }\end{array}$ & $\begin{array}{l}\text { RAS- } 41 \\
\text { (Punt. } 5 \text { factores) } \\
\text { IPII, MAS-A, } \\
\text { PANSS }\end{array}$ & $\begin{array}{l}\text { Correlación entre RAS-Esperanza y } \\
\text { PANSS- Positiva }(\mathrm{r}=-0.34) \text {, -Negativa } \\
(\mathrm{r}=-0.38) \text { y }- \text { Malestar emocional } \\
(\mathrm{r}=-0.38) \text {. } \\
\text { Correlación entre RAS-Ayuda y } \\
\text { PANSS- Negativa }(r=-0.33) \text {. } \\
\text { Correlación entre RAS-Metas y } \\
\text { PANSS- Hostilidad }(r=0.35) \text {. } \\
\text { Correlación entre RAS-No dominación } \\
\text { y PANSS-Hostilidad }(r=-0.31) \text { y } \\
\text {-Malestar emocional }(r=-0.35) \text {. } \\
\text { Relación entre RAS-No Dominación y } \\
\text { MAS-A-Autorreflexión }(F=4.16) \text {. } \\
\text { Relación entre Descentramiento con } \\
\text { RAS-Esperanza }(F=5.32), \text { RAS-Ayuda } \\
(F=4.42) \text { y RAS-Otras }(F=4.14)\end{array}$ & $\begin{array}{l}\text { Relación entre algunos factores del } \\
\text { RAS y parte de la sintomatología. } \\
\text { Relación entre algunos factores del } \\
\text { RAS con habilidades metacognitivas } \\
\text { para pensar sobre uno mismo. }\end{array}$ \\
\hline
\end{tabular}

Nota: TMG: Trastorno Mental Grave; ESQ: Esquizofrenia o trastorno esquizoafectivo; Corr.: Correlacional; Regr.: Modelo de Regresión múltiple; Punt.: Puntuación; AQ-27: Attribution Questionnaire; BASIS-32: Behaviour and Symptom Identification Scale; BDI-II: Beck Depression Inventory-Revised; BUS: Boston University Empowerment Scale; CANSAS: Camberwell Assessment of Need Short Appraisal Schedule; CDSS: Calgary Depression Scale for Schizophrenia; CES-D : Center for Epidemiological Studies-Depression Scale; CIM: Community Integration Measure; EDD: Direct discrimination experiences; ES: Empowerment Scale; GAF: Global Assessment of Functioning; HHI: Herth Hope Index; HoNOS: Health of the Nation Outcome Scales; IPII: Indiana Psychiatric Illness Interview; IPQS: Illness Perception Questionnaire for Schizophrenia; ISMI: Internalized Stigma of Mental Illness Scale; K-10: Kessler-10; LQLQP: Lancaster Questionnaire Life Quality Profile; LSP-16: Life Skills Profile-16; MANSA: Manchester Short Assessment of Quality of Life; MAS-A: Abbreviated Metacognition Assessment Scale; MHRM: Mental Health Recovery Measure; MLS: Meaning of Life Subscale; MSAQL: Manchester Short Assessment of Quality of Life; MSPSS: Multidimensional Scale of Perceived Social Support; NARA: Needs and Resources Assessment; PANSS: Positive and Negative Syndrome Scale; PDD: Perceived Devaluation and Discrimination Scale; PPS: Participation in peer support; QOLI: Quality of Life Interview; RAS: Recovery assessment Scale; RSES: Rosenberg Self-esteem Scale; SELS-SV: Social and emotional Loneliness Scale-short version; SFS: Social Functioning Scale; SF-12: MCS and PCS: Mental Health Composite and Physical Health Composite; SISR: Self-Identified Stage of Recovery; SNS: Social Network Scale; SRM: Stages of Recovery Measure; SSQ: Social Support Questionnaire; SUMD: Scale to assess Unawareness of Mental Disorder; SWN-K: Subjective Well-being under Neuroleptic treatment scale; TMG: trastornos mentales graves; WQLQ: Wisconsin Quality of Life Questionnaire.

de las intervenciones. La Tabla 1 resume algunos de los principales trabajos en este ámbito. Así diversos estudios han abordado la descripción de la relación entre recuperación subjetiva y otras variables clínicas y psicosociales mediante el análisis de correlaciones. Los trabajos que han empleado el RAS (Corrigan et al., 1999) como medida de recuperación subjetiva han reportado relaciones entre este instrumento y un gran número de variables como sintomatología clínica, funcionamiento social, autoestima, soporte social, edad, participación en actividades, necesidades percibidas, estigma social e internalizado, soledad, empoderamiento, esperanza, ayuda mutua, resiliencia, calidad de vida o habilidades metacognitivas (ver Tabla 1).

Estas investigaciones han estudiado la recuperación subjetiva fundamentalmente en personas con trastornos mentales graves (TMG) aunque otras se han centrado en personas con esquizofrenia o trastornos esquizoafectivos. A este respecto cuando se ha comparado la recuperación subjetiva entre los diferentes diagnósticos se ha observado que las personas con trastorno afectivo bipolar presentan una mayor percepción de recuperación que las personas con esquizofrenia (Lloyd, King y Moore, 2009). Sin embargo el empleo de una metodología correlacional bivariada aplicada al estudio de las relaciones entre constructos psicológicos complejos determinados por múltiples factores, presenta limitaciones a la hora de establecer cuál es la contribución única de las variables objeto de análisis, independientemente de la varianza compartida entre ellas. Por ejemplo, en el estudio de Corrigan et al., (1999), pese a la correlación identificada entre recuperación subjetiva y calidad de vida, 
cuando esta variable se incluyó en un modelo de regresión múltiple junto a otras, la calidad de vida dejó de mostrar una contribución significativa en la predicción de recuperación. En este sentido alternativas analíticas como el análisis de regresión múltiple o los modelos de ecuaciones estructurales permiten establecer la contribución única de determinadas variables psicológicas sobre otras, aislando la cantidad de varianza compartida entre ellas al permitir la inclusión de conjuntos de variables más amplios. Pese al interés teórico y aplicado de las aproximaciones multivariadas al estudio de las variables implicadas en el proceso de recuperación subjetiva, son escasas las investigaciones que la han empleado a tal fin. Así sólo ocho de las 17 investigaciones revisadas emplearon la metodología de la regresión múltiple y dos más ecuaciones estructurales. Estos trabajos han estudiado la contribución a las expectativas de recuperación de tres tipos de variables: sociodemográficas, de recuperación clínica y psicológicas. En primer lugar las variables sociodemográficas como el género y la edad demostraron tener poca capacidad predictiva en las expectativas de recuperación (Corrigan et al, 1999; Corrigan, 2006). De hecho en la investigación de Corrigan et al., (1999), los propios autores recomiendan el estudio en profundidad de dicha relación debido a ciertos problemas metodológicos de su diseño. En un trabajo posterior del mismo grupo, la edad explicó una escasa porción de la varianza del RAS (Corrigan, 2006). Junto a su escasa capacidad predictiva y la ausencia de relación entre edad y RAS en otros estudios (Hendryx, Green y Perrin, 2009; Roe et al., 2011), se podría indicar que la aportación de esta variable a la predicción de la recuperación subjetiva es en el mejor de los casos anecdótica. En segundo lugar las variables de recuperación clínica, es decir la sintomatología y el funcionamiento social, revelaron capacidad predictiva en la recuperación subjetiva aunque no mostraron ser predictores consistentes en otros trabajos revisados. Así mientras algunos factores de la Escala de Evaluación de la Recuperación (RAS, Corrigan et al., 1999) han sido predichos por los síntomas psiquiátricos y la participación en actividades (Corrigan et al., 2004; Hendryx et al., 1999;) otros estudios no encontraron tal relación (Corrigan et al., 1999; Roe, Mashiach-Eizenberg, \& Corrigan, 2012). En muchos trabajos la sintomatología perdió su capacidad predictiva cuando se consideraron otras variables psicológicas como la autoestima (Corrigan et al., 1999). De hecho algunas investigaciones han revelado falta de consistencia en la relación de uno de los criterios operativos del funcionamiento social, i.e., tener un empleo, con una mayor recuperación subjetiva (Connell, King y Crowe, 2011; Lloyd et al., 2009). Los estudios que han utilizado la metodología de ecuaciones estructurales han mostrado que la recuperación subjetiva podría tener un papel mediador en resultados clínicos y funcionales en las personas con TMG. Así en el trabajo de Muñoz, Sanz, Pérez-Santos y Quiroga, (2010) la recuperación subjetiva junto al estigma internalizado predijeron directamente resultados conductuales como el funcionamiento social y la autonomía personal. Este efecto moderador de la recuperación subjetiva también se observó en el estudio de Cavelti, Beck, Kvrgic, Kossowsky y Vauth (2012), donde la asociación existente entre una mayor conciencia de enfermedad y el aumento de los síntomas depresivos se debilitó ante una mejor percepción subjetiva de recuperación. En tercer lugar variables psicológicas como la autoestima, el empoderamiento, la esperanza, la calidad de vida, la percepción de apoyo social y la soledad, aparecen como importantes predictores de recuperación subjetiva (Cavelti et al., 2012; Corrigan et al., 1999; Corrigan y Phelan, 2004; Hendryx et al., 2009; Roe et al., 2011; Roe et al., 2012). La autoestima mostró ser uno de los predictores de recuperación subjetiva en el estudio de validación del RAS (Corrigan et al., 1999), si bien la relación entre autoestima y recuperación subjetiva podría ser bidireccional. Así en el trabajo de Mukolo, Heflinger y Baxter, (2011) la autoestima fue predicha por una mayor recuperación subjetiva concretamente los elementos vinculados al mantenimiento de la esperanza y la predisposición a pedir ayuda (Mukolo et al., 2011). El empoderamiento aparece relacionado con la recuperación subjetiva en tres estudios, pero solo en uno de ellos demostró capacidad predictiva (Corrigan et al., 1999). Igualmente la esperanza se relacionó con recuperación en dos estudios, pero solo tuvo capacidad predictiva en uno de ellos (Corrigan et al., 2004). Una mayor calidad de vida se relacionó con mayor recuperación subjetiva en tres trabajos pero solo predijo recuperación subjetiva en dos trabajos (Hendryx et al, 2009; Roe et al., 2012). Por último la percepción de tener un adecuado soporte social o la soledad percibida aparecen como importantes predictores de la recuperación subjetiva (Cavelti et al., 2012; Hendrix et al., 2009; Roe et al., 2011; Roe et al., 2012). En conclusión los estudios revisados destacan la contribución de ciertas variables en la predicción de mayores expectativas de recuperación en la esquizofrenia y otros TMG. Entre estos predictores parecen jugar un papel destacado aspectos psicológicos como la autoestima, la esperanza, la soledad, el empoderamiento y la percepción de apoyo social y en menor medida la sintomatología y el funcionamiento social. Pese a la relevancia de estos hallazgos todavía son escasos los datos disponibles y el número de variables exploradas. De hecho existen evidencias indirectas de que dos variables aún no explo- 
radas de manera conjunta podrían contribuir a la predicción de la recuperación subjetiva, tal y como se indica en la sección siguiente.

Una las variables que podría resultar de importancia en la predicción de la recuperación subjetiva sería el estigma internalizado, definido como un proceso subjetivo caracterizado por la aprobación de los estereotipos sobre los trastornos mentales, sentimientos negativos hacia uno mismo y comportamientos desadaptativos de autodiscriminación (Livingston y Boyd, 2010). Revisiones recientes han relacionado el estigma internalizado con la esperanza, la autoestima y el empoderamiento (Livingston y Boyd, 2010). Más concretamente, en el estudio de validación de la Escala de Estigma Internalizado de Enfermedad Mental (Internalized Stigma of Mental Illness, ISMI; Ritsher, Otilingam y Grajales, 2003), los autores hallaron una correlación positiva entre estigma internalizado y recuperación subjetiva. Posteriormente el estudio de Muñoz et al., (2010), evaluando un modelo empírico sobre estigma internalizado, confirmó la relación positiva entre ambas variables. Pese a estas relaciones y a las elevadas tasas de prevalencia del estigma internalizado en esquizofrenia, de más de un tercio según los estudios (Gerlinger et al., 2013), no se ha considerado el potencial papel de esta variable en la predicción de la recuperación subjetiva. Otra de las variables no estudiadas en trabajos previos son las estrategias de afrontamiento, definidas como los esfuerzos cognitivos y conductuales que desarrollan los individuos para manejar las demandas externas y/o internas que son evaluadas como desbordantes respecto a los recursos personales (Lazarus y Folkman, 1986). Pese a que las estrategias de afrontamiento se han relacionado con aspectos estrechamente vinculados con la recuperación subjetiva, como la autoestima, la esperanza y el empoderamiento (Rüsch et al., 2009; Vauth, Kleim, Wirtz y Corrigan, 2007; Yanos, Roe, Markus y Lysaker, 2008;), ningún estudio hasta la fecha ha considerado simultáneamente las estrategias de afrontamiento y las expectativas de recuperación. En resumen, y pese a que tanto el estigma internalizado como las estrategias de afrontamiento podrían contribuir a determinar la visión que las personas con esquizofrenia tienen de su propio proceso de recuperación, hasta la fecha ningún estudio ha examinado de forma conjunta su aportación a la predicción de la recuperación subjetiva.

El objetivo del presente estudio fue explorar la capacidad predictiva conjunta de variables previamente consideradas relevantes y otras nuevas respecto a la experiencia subjetiva de recuperación en personas con esquizofrenia, clarificando ciertas inconsistencias de la literatura previa. De acuerdo a las evidencias indirectas existentes (Muñoz et al., 2010; Ritsher et al., 2003;
Rüsch et al., 2009; Vauth et al., 2007; Yanos et al., 2008) se planteó como hipótesis que si la experiencia subjetiva de recuperación en personas con esquizofrenia está determinada por variables estratégicas no consideradas conjuntamente con anterioridad, como son las estrategias de afrontamiento y la internalización del estigma, entonces dichas variables mostrarían capacidad predictiva sobre las expectativas de recuperación.

\section{Método}

Los datos de la presente investigación fueron obtenidos de un estudio descriptivo llevado a cabo en los cinco Centros de Rehabilitación Psicosocial y Laboral (CRPSL) existentes en la provincia de Toledo siendo recursos especializados en la atención psicosocial a personas con trastornos mentales graves.

\section{Participantes}

La muestra estuvo formada por 43 pacientes con diagnóstico de esquizofrenia atendidas en los CRPSL de la provincia de Toledo. Todas las personas fueron diagnosticadas de esquizofrenia previamente a la derivación al centro por su facultativo de referencia (psiquiatra o psicólogo clínico) desde las Unidades de Salud Mental. Para la selección de los participantes los psicólogos de cada CRPSL tuvieron en cuenta los siguientes criterios de inclusión: (1) diagnóstico principal de esquizofrenia; (2) ser atendido en uno de los cinco CRPSL de la provincia de Toledo; (3) edad comprendida entre los 18 y 50 años; (4) ausencia de deterioro cognitivo grave (según el Mini-examen del Estado Mental Modificado, 3MS; Teng y Chui, 1987); (5) ausencia de diagnóstico de retraso mental; (6) ausencia de comorbilidad con un diagnóstico de trastorno por dependencia de sustancias; (7) ausencia de síntomas psicóticos agudos que impidan la cumplimentación de los instrumentos en el momento del estudio (según la Escala del Síndrome Positivo y Negativo, PANSS; Kay, Fiszbein y Opler, 1987) y (8) en el caso de personas atendidas en su domicilio, que aceptaran acudir al CRPSL. Fruto de la aplicación de estos criterios se seleccionaron 70 personas de las que 43 accedieron a participar (11 mujeres) con una edad media de $36.7 \pm$ 8.1 años. Los participantes fueron diagnosticados de esquizofrenia con una media de edad de $24.3 \pm 6.6$ años, una duración media del trastorno de $13.4 \pm 7.6$ años y una media de $2.7 \pm 3.3$ hospitalizaciones por motivos psiquiátricos antes de iniciar su participación en el estudio. Los participantes no presentaron comorbilidad con otros trastornos mentales, excepto uno de ellos que presentaba como diagnóstico secundario Trastorno de la 
Personalidad sin especificar. Se evaluó el funcionamiento cognitivo con el Mini-Examen del Estado Mental Modificado (3MS; Teng y Chui, 1987) mostrando una puntuación media de $94.2 \pm 5.1$ (rango de 0 a 100 donde a mayor puntuación mejor funcionamiento cognitivo). La Tabla 2 resume las principales características clínicas y sociodemográficas de los participantes. En el momento del estudio todos los participantes estaban recibiendo tratamiento farmacológico y rehabilitador.

Tabla 2. Características clínicas y sociodemográficas de los participantes

\begin{tabular}{lr}
\hline \multicolumn{1}{c}{ Características } & $\mathrm{N}(\%)$ \\
\hline Diagnóstico & \\
Esquizofrenia paranoide & $33(76.7)$ \\
Esquizofrenia hebrefrénica & $2(4.6)$ \\
Esquizofrenia indiferenciada & $3(7)$ \\
Esquizofrenia residual & $2(4.6)$ \\
Esquizofrenia simple & $3(7)$ \\
Clasificación diagnóstica & \\
CIE-9 & $1(2.3)$ \\
CIE-10 & $13(30.2)$ \\
DSM-IV & $5(11.6)$ \\
No codificado en derivación a CRPSL & $17(39.5)$ \\
Se desconoce & $7(16.3)$ \\
Fármacos & \\
Solo antipsicóticos & $5(11.6)$ \\
Antipsicóticos y otros & $38(88.4)$ \\
Residencia & \\
Rural & \\
Semirural & $20(46.5)$ \\
Urbana & $11(25.6)$ \\
Estado civil & $12(27.9)$ \\
Soltero & \\
Casado & $17(32.6)$ \\
Separado/divorciado & $19(13.9)$ \\
Nivel de estudios & $19(44.2)$ \\
Sin educación primaria & $2(4.6)$ \\
Educación primaria & \\
Educación secundaria & \\
Universitario & \\
Situación laboral & \\
\hline
\end{tabular}

\section{Instrumentos}

Escala de Evaluación de la Recuperación (Recovery Assessment Scale, RAS; Corrigan et al., 1999). Esta escala evalúa las expectativas de recuperación y consta de
41 ítems valorados en una escala del 1 al 5 (muy en desacuerdo-muy de acuerdo) que son agrupados en cinco factores: Confianza personal y esperanza, Disposición a pedir ayuda, Orientación hacia las metas y el éxito, Confianza en los otros y No dominación por los síntomas (Corrigan et al., 2004). Se utilizó una versión breve de 18 ítems (RAS-18) adaptada la castellano ad hoc para el presente estudio (método de traducción-retrotraducción), seleccionando un número equilibrado de los ítems más representativos de cada factor a partir de los datos originales de validación (ver Tabla 3; Corrigan et al., 2004). La puntuación en la escala breve comprendió un rango entre 18 y 90 donde a mayor puntuación mejores expectativas de recuperación $(\alpha$ de Cronbach $=0.81)$.

Escala de Estigma Internalizado de Enfermedad Mental (Internalized Stigma of Mental Illness, ISMI; Ritsher et al., 2003). Esta escala evalúa las dimensiones cognitivas, emocionales y conductuales del estigma internalizado, la discriminación experimentada debido a la enfermedad mental, así como la capacidad para resistir al estigma. Se utilizó la adaptación al castellano por Brohan, Elgie, Sartorius y Thornicroft (2010). La escala incluye 29 ítems que son valorados del 1 al 4 (totalmente de acuerdo-totalmente en desacuerdo). La puntuación final en la escala comprendió un rango entre 1 y 4 donde a mayor puntuación mayor estigma internalizado ( $\alpha$ de Cronbach $=0.87$ ).

Escala de Autoestima de Rosenberg (Rosenberg Self-Esteem Scale, RSE; Rosenberg, 1965). Esta escala evalúa los sentimientos de respeto y aceptación de sí mismo. Se utilizó la validación realizada en población española que ha mostrado una alta consistencia interna, fiabilidad y validez de constructo (Vázquez-Morejón, Jiménez y Vázquez-Morejón, 2004). La escala incluye diez ítems que son valorados de 1 a 4 (totalmente de acuerdo-totalmente en desacuerdo). La puntuación comprendió un rango entre 10 y 40 donde a mayor puntuación menor autoestima ( $\alpha$ de Cronbach $=0.78)$.

Inventario de Estrategias de Afrontamiento (Coping Strategies Inventory, CSI; Tobin, Holroyd, Reynolds, y Kigal, 1989). El instrumento incluye 41 ítems que son valorados de 0 a 5 (en absoluto-totalmente) y agrupados en dos subescalas: Manejo Adecuado que evalúa estrategias activas y adaptativas para compensar la situación estresante (resolución de problemas, reestructuración cognitiva, expresión emocional, apoyo social) y Manejo Inadecuado que evalúa estrategias pasivas y desadaptativas (pensamiento desiderativo, autocrítica, evitación de problemas y retirada social). Se utilizó la versión del CSI validada en población española que ha mostrado excelentes propiedades psicométricas superando incluso las del estudio original (Cano García, Rodríguez Franco, 
Tabla 3. Ítems y factores del RAS-18

\begin{tabular}{ll}
\hline \multicolumn{1}{c}{ Factor } & \multicolumn{1}{c}{ Ítems originales del RAS-41 } \\
\hline Orientación hacia las metas y el éxito & 2. Tengo mi propio plan sobre como estar o llegar a estar bien. \\
& 3. Tengo objetivos en la vida que quiero alcanzar. \\
& 4. Creo que puedo alcanzar mis actuales objetivos personales. \\
& 5. Mi vida tiene un sentido. \\
Confianza personal y esperanza & 14. Puedo manejar lo que sucede en mi vida. \\
& 15. Me gusto a mí mismo. \\
& 22. Algo bueno sucederá en algún momento. \\
& 24. Tengo esperanzas sobre mi futuro. \\
& 27. Afrontar mi enfermedad mental ha dejado de ser el centro de mi vida. \\
28. Los síntomas de la enfermedad interfieren cada vez menos en mi vida. & 29. Cuando aparecen, mis síntomas son un problema que dura cada vez menos. \\
No dominación por los síntomas & 30. Sé cuándo pedir ayuda. \\
3isposición a pedir ayuda & 32. Pido ayuda cuando lo necesito. \\
6. Incluso cuando no me cuido a mi mismo, otras personas lo hacen por mí. & 37. Tengo gente con la que puedo contar. \\
39. Incluso cuando no creo en mi mismo otra gente cree en mí. & 40. Es importante tener variedad de amigos. \\
\hline
\end{tabular}

y García Martínez, 2007). La puntuación en cada subescala comprendió un rango entre 0 y 100 donde a mayor puntuación mayor uso de estrategias de afrontamiento $(\alpha$ de Cronbach $=0.84$ )

Escala del Sindrome Positivo y Negativo (The Positive and Negative Síndrome Scale, PANSS; Kay et al., 1987). La escala consta de 30 ítems que son valorados de 1 a 7 (ausente-extremo) y agrupados en tres subescalas (Positiva, Negativa y General). Se utilizó la versión española de la PANSS validada en población española que ha demostrado propiedades psicométricas similares a la escala original con elevada fiabilidad interobservador, validez de constructo y de criterio (Peralta y Cuesta, 1994). La puntuación de las dos primeras escalas comprendió un rango entre 7 y 49 y la última entre 16 y 112 donde a mayor puntuación mayor presencia y gravedad de la sintomatología. En el presente estudio se consideró como marco temporal la semana previa a la aplicación del protocolo de evaluación $(\alpha$ de Cronbach $=0.84)$.

Escala de Funcionamiento Social (Social Functioning Scale, SFS; Birchwood, Smith, Cochrane, Wetton y Copestake, 1990) La escala consta de siete subescalas: Aislamiento, Comunicación interpersonal, Independencia-ejecución, Independencia-competencia, Actividades del tiempo libre, Conducta prosocial y Empleo-ocupación. Se utilizó la versión de la SFS validada en población española que ha mostrado propiedades psicométricas adecuadas (Torres y Olivares, 2005). En la presente investi- gación se consideraron las puntuaciones de todas las subescalas, excepto la última de Empleo-ocupación comprendiendo un rango entre 0 y 219 donde a mayor puntuación mejor funcionamiento social $(\alpha$ de Cronbach $=0.86$ ).

\section{Procedimiento}

Los participantes fueron evaluados en dos sesiones diferentes de 60 minutos de duración cada una en las que los instrumentos fueron aplicados por psicólogos expertos. Antes de la primera sesión todos los participantes fueron informados sobre el propósito de la investigación y dieron su consentimiento a participar por escrito, de acuerdo con la Declaración de Helsinki. En la primera sesión se evaluó la sintomatología y el funcionamiento social, mientras que en la segunda se evaluó recuperación subjetiva, estigma internalizado, autoestima y estrategias de afrontamiento. Ambas sesiones se realizaron en el horario habitual de asistencia al centro.

\section{Resultados}

\section{Correlaciones entre recuperación subjetiva y variables clínicas y psicosociales}

Las Tablas 4 y 5 resumen los estadísticos descriptivos de todas las medidas registradas y los coeficientes de correlación de Pearson obtenidos entre las variables, res- 
pectivamente. Como muestra la Tabla 5 se hallaron correlaciones significativas negativas entre el RAS y el ISMI, y entre el RAS y la subescala del síndrome negativo de la PANSS. Por otro lado se hallaron correlaciones significativas positivas entre el RAS y la subescala Manejo Adecuado del CSI, la RSE y el SFS. No se encontraron otras correlaciones significativas entre el RAS y medidas como la subescala Manejo Inadecuado del CSI, la subescala positiva y general de la PANSS, ni con el resto de variables clínicas o sociodemográficas evaluadas.

Tabla 4. Estadísticos descriptivos $(\mathrm{N}=43)$ de las variables

\begin{tabular}{lrr}
\hline \multicolumn{1}{c}{ Variables } & Media (D.T.) & \multicolumn{1}{c}{ Rango } \\
\hline RAS-18 & $68.4(7.9)$ & $18-90$ \\
ISMI & $2.1(0.4)$ & $1-4$ \\
PANSS Positiva & $11.1(4.1)$ & $7-49$ \\
PANSS Negativa & $13.9(6.3)$ & $7-49$ \\
PANSS General & $26.5(6.7)$ & $16-112$ \\
RSE & $21(4.2)$ & $10-40$ \\
SFS & $118.1(19.3)$ & $0-219$ \\
CSI Adecuado & $33.4(16.2)$ & $0-100$ \\
CSI Inadecuado & $26.1(8)$ & $0-100$ \\
\hline
\end{tabular}

Nota: RAS-18: Versión breve de 18 ítems de la Escala de Evaluación de la Recuperación; ISMI: Escala de Estigma Internalizado en Enfermedad Mental; PANSS: Escala del Síndrome Positivo y Negativo; RSE: Escala de Autoestima de Rosenberg; SFS: Escala de Funcionamiento Social; CSI: Inventario de Estrategias de Afrontamiento.

\section{Predictores de la recuperación subjetiva}

Las variables que mostraron una correlación significativa con el RAS fueron seleccionadas como predicto- res en el análisis de regresión múltiple tomando la recuperación subjetiva como variable criterio. Así se incluyeron ISMI, la subescala Manejo Adecuado del CSI, RSE, SFS y la subescala del Síndrome Negativo de la PANSS. El modelo alcanzado fue significativo $\left(R^{2} \mathrm{co}-\right.$ rregida $=0.551 ; F=12.5, p<0.0001)$ y mostró que la subescala Manejo Adecuado del CSI, el ISMI y el RSES tuvieron una contribución única significativa del 15,3\%, $12,3 \%$ y $11 \%$ a la puntuación total del RAS, respectivamente (ver Tabla 6). Sin embargo ni el SFS ni la subescala del Síndrome Negativo de la PANSS mostraron una contribución significativa en la predicción de la puntuación total del RAS. El modelo de regresión puede ser generalizado ya que se ajustó razonablemente bien a los supuestos de la prueba. Específicamente los valores de tolerancia fueron superiores a 0.2 , ningún $F I V$ superó los 10 puntos y los residuos no estuvieron correlacionados entre si (Durbin-Watson $=2.4)$.

\section{Discusión}

El objetivo del presente estudio fue clarificar la contribución de un conjunto de variables clínicas y psicosociales a la predicción de la recuperación subjetiva en personas con diagnóstico de esquizofrenia. Dicha información resulta relevante tanto para la elaboración de modelos teóricos sobre la recuperación subjetiva como para el desarrollo de intervenciones que fomenten mejores expectativas de recuperación en esta población. Para ello se analizó la contribución de las variables afrontamiento, estigma internalizado, autoestima, sintomatología y funcionamiento psicosocial en la predicción de la recuperación subjetiva mediante la metodología del análisis de regresión múltiple. Teniendo en cuenta los ha-

Tabla 5. Correlaciones entre las variables

\begin{tabular}{|c|c|c|c|c|c|c|c|c|c|}
\hline & 1 & 2 & 3 & 4 & 5 & 6 & 7 & 8 & 9 \\
\hline 1. RAS-18 & 1 & & & & & & & & \\
\hline 2. ISMI & $-0.57^{* *}$ & 1 & & & & & & & \\
\hline 3. CSI Adecuado & $0.54 * *$ & $-0.33^{*}$ & 1 & & & & & & \\
\hline 4. CSI Inadecuado & -0.05 & -0.36 & 0.02 & 1 & & & & & \\
\hline 5. RSE & $0.51 * *$ & $-0.47 * *$ & 0.06 & 0.16 & 1 & & & & \\
\hline 6. SFS & $0.49^{* *}$ & -0.19 & $0.36^{*}$ & -0.04 & $0.32 *$ & 1 & & & \\
\hline 7. PANSS Positiva & 0.02 & 0.13 & 0.25 & 0.14 & 0.05 & 0.21 & 1 & & \\
\hline 8. PANSS Negativa & $-0.33^{*}$ & 0.05 & $-0.4 * *$ & -0.12 & -0.14 & $-0.43^{* *}$ & -0.01 & 1 & \\
\hline 9. PANSS General & -0.16 & 0.14 & 0.05 & -0.1 & 0.17 & -0.01 & $0.41 * *$ & $0.52 * *$ & 1 \\
\hline
\end{tabular}

Nota: RAS-18: Versión breve de 18 ítems de la Escala de Evaluación de la Recuperación; ISMI: Escala de Estigma Internalizado de Enfermedad Mental; CSI: Inventario de Estrategias de Afrontamiento; RSE: Escala de Autoestima de Rosenberg; SFS: Escala de Funcionamiento Social; PANSS: Escala del Síndrome Positivo y Negativo. ${ }^{*} p<0.05$; $* * p<0.01$. 
Tabla 6. Resultados del análisis de regresión múltiple sobre la variable dependiente RAS-18

\begin{tabular}{lccccc}
\hline \multicolumn{1}{c}{ Variables predictoras } & $B$ & $E . T(B)$ & $\beta$ & $p$ & 0.391 \\
\hline CSI Adecuado & 0.16 & 0.06 & 0.32 & 0.014 & 0.338 \\
RSE & 0.51 & 0.23 & 0.27 & 0.036 & -0.358 \\
ISMI & -6.34 & 2.72 & -0.29 & 0.025 & 0.269 \\
SFS & 0.07 & 0.05 & 0.21 & 0.097 & -0.078 \\
PANSS Negativa & -0.07 & 0.15 & -0.06 & 0.636 & \\
\hline
\end{tabular}

Nota: $R^{2}$ corregida $=0.551(F=12.5, p<0.0001)$. RAS-18: Versión breve 18 ítems Escala de Evaluación de la Recuperación; CSI Adaptativas: Inventario de Estrategias de Afrontamiento, Subescala estrategias adaptativas; RSE: Escala de Autoestima de Rosenberg; ISMI: Escala de Estigma Internalizado de Enfermedad Mental; SFS: Escala de Funcionamiento Social; PANSS Negativa: Escala del Síndrome Positivo y Negativo.

llazgos de trabajos previos se planteó que el estigma internalizado y las estrategias de afrontamiento tendrían un papel destacado en la predicción de la recuperación subjetiva (Muñoz et al., 2010; Ritsher et al., 2003; Rüsch et al., 2009; Vauth et al., 2007; Yanos et al., 2008)

Las variables que predijeron de forma significativa la recuperación subjetiva fueron las estrategias adaptativas de afrontamiento, el estigma internalizado y la autoestima. En primer lugar las estrategias adaptativas de afrontamiento fue la dimensión que más contribuyó a la predicción de la recuperación subjetiva. Dichas estrategias incluyen diferentes habilidades utilizadas por la persona para manejar de forma activa las situaciones estresantes tales como la solución de problemas, la reestructuración cognitiva, el soporte social y la expresión emocional. Mientras la solución de problemas y la reestructuración cognitiva serían estrategias centradas en el problema dirigidas a modificar la situación que produce estrés y el significado de dicha situación, la expresión emocional y el soporte social resultarían estrategias centradas en la emoción dirigidas a la búsqueda de apoyo emocional y la manifestación de las emociones producidas por una situación estresante (Cano et al., 2007). Algunos estudios previos con el mismo tipo de población encontraron relación entre el empleo de estrategias de afrontamiento pasivas y la disminución de la esperanza, estando esta última estrechamente vinculada a la recuperación subjetiva (Rüsch et al., 2009; Yanos et al., 2008). Sin embargo nuestros resultados sugieren de manera innovadora un papel importante de las estrategias adaptativas de afrontamiento en la predicción de la recuperación subjetiva. El estigma internalizado fue la segunda dimensión en importancia que contribuyó a explicar la recuperación subjetiva. Aunque la relación entre el estigma internalizado y las expectativas de recuperación ha sido descrita previamente (Muñoz et al., 2010; Ritsher et al., 2003), los presentes resultados demuestran la capacidad predictiva de esta variable en la recuperación subjetiva. En el modelo sobre estigma internalizado de Muñoz et al.,
(2010) los autores sugieren que el estigma social, y no el internalizado, explica la recuperación subjetiva. Sin embargo la alta correlación entre ambos tipos de estigma pudo enmascarar en su modelo de ecuaciones estructurales la relación entre estigma internalizado y recuperación subjetiva, quedando reflejada únicamente la contribución de la variable que más varianza explicó, i.e., estigma social. El valor predictivo del estigma internalizado descrito en el presente estudio es coherente con los datos que sugieren que la recuperación implica una resistencia a la estigmatización (Sibitz, Unger, Woppmann, Zidek y Amering, 2009). En este sentido una alta internalización del estigma potenciaría la falta de esperanza en el futuro y en las posibilidades de recuperación (Lysaker, Roe y Yanos, 2007; Yanos et al., 2008). Por último la autoestima fue la tercera dimensión en orden de importancia que contribuyó a la predicción de la recuperación subjetiva en consonancia con un estudio predictivo previo. Pese a que este hallazgo ya fue señalado en uno de los primeros trabajos sobre predictores de expectativas de recuperación (Corrigan et al., 1999), la contribución de esta variable ha sido ignorada en trabajos posteriores (ver Tabla 1). Los resultados del presente trabajo refuerzan asimismo los datos de otros estudios cualitativos en los que se identifica una identidad positiva y una valoración de las propias capacidades como marcadores fundamentales de recuperación (Leamy et al., 2011).

Dos de los indicadores objetivos de recuperación estudiados, i.e., funcionamiento social y sintomatología negativa, mostraron relación con la recuperación subjetiva, si bien no se mantuvieron como predictores de ésta cuando se consideraron de manera conjunta con el resto de variables evaluadas. Existe controversia en la literatura sobre el papel del funcionamiento psicosocial en la recuperación subjetiva. Mientras que algunos estudios previos han encontrado relación entre ellas (Chiba et al., 2010; Muñoz et al., 2010), otros estudios no han encontrado tal relación (Andresen et al., 2010; Roe et al., 
2011). A este respecto el análisis de regresión ha contribuido en el pasado y en el presente trabajo a clarificar esta aparente inconsistencia. Así mientras ciertos aspectos vinculados al funcionamiento social como la participación en actividades ha revelado capacidad predictiva sobre el RAS (Hendryx et al., 2009), el funcionamiento social de forma global no resultó ser un buen predictor de recuperación subjetiva (Roe et al., 2012). Igualmente el papel de la sintomatología en la recuperación subjetiva ha suscitado controversias con anterioridad. Así algunos estudios previos encontraron relación entre sintomatología con la puntuación total del RAS (Andresen et al., 2010; Corrigan et al., 1999) o relaciones parciales con algunos de sus factores (Kukla, Lysaker y Salyers, 2013; McNaught et al., 2007; Roe et al., 2011). Incluso ciertos factores del RAS resultaron moderadores en la relación entre conciencia de enfermedad y síntomas depresivos en personas con esquizofrenia (Cavelti et al., 2012). Sin embargo otros trabajos empleando regresión múltiple, o bien han demostrado una ausencia de capacidad predictiva de la sintomatología en la recuperación subjetiva (Corrigan et al., 1999), o bien dicha sintomatología solo ha predicho alguno de los factores que componen las escalas de recuperación como el RAS (Corrigan et al., 2004). En conjunto los resultados de las diferentes investigaciones existentes resaltan la complejidad de la relación entre sintomatología y recuperación subjetiva, apuntando que pese a la posible asociación entre ambas, la sintomatología no parece determinante en la predicción de la recuperación subjetiva. Concretamente los datos del presente estudio muestran que mientras la sintomatología negativa tuvo relación con la recuperación subjetiva, cuando las estrategias de afrontamiento se incluyeron en el modelo de regresión, los síntomas negativos no predijeron dicha recuperación subjetiva. En otras palabras, las personas avalarían su experiencia de recuperación, no tanto cuando hubieran desaparecido sus síntomas, sino cuando hubieran adquirido control o estrategias para el manejo de los mismos, enfatizando la importancia del afrontamiento en el proceso de recuperación. La incapacidad del funcionamiento social y la sintomatología para predecir la recuperación subjetiva en el presente estudio apoya las investigaciones que afirman que los indicadores objetivos y subjetivos de recuperación se encuentran relacionados pero son relativamente independientes (Roe et al., 2011). Ambas definiciones de recuperación, clínica y subjetiva, serían perspectivas independientes y complementarias en el abordaje de la recuperación (Silverstein y Bellack, 2008) y por tanto deben ser valoradas de manera separada en los servicios de atención a las personas con esquizofrenia.
El trabajo actual presenta ciertas limitaciones que deben ser consideradas de cara a futuras investigaciones. En primer lugar, y pese a que la muestra empleada resulta adecuada para los objetivos de análisis de este estudio, sería deseable replicar los presentes resultados con muestras de mayor tamaño a fin de garantizar la representatividad de los datos obtenidos. En segundo lugar, debe observarse que los participantes mostraron una buena adherencia al tratamiento y una baja severidad sintomatológica (ver Tabla 4). A este respecto, personas con baja adherencia al tratamiento o sintomatología de mayor gravedad podrían manifestar un mayor estigma internalizado o utilizar diferentes estrategias de afrontamiento (Fernández y Díaz, 2001; Fung, Tsang y Corrigan, 2008).

En conclusión, las estrategias adaptativas de afrontamiento, el estigma internalizado y la autoestima deben ser considerados como objetivos relevantes en la promoción de la recuperación subjetiva en personas con esquizofrenia. Específicamente cabría recomendar por un lado la conveniencia de incluir alguna de las medidas disponibles de evaluación de la recuperación subjetiva en los servicios de atención a las personas con esquizofrenia. Estas nuevas medidas complementarían las medidas tradicionales de recuperación, es decir las que evalúan la sintomatología y el funcionamiento social. Por otro lado, y en relación con los objetivos de intervención, sería necesario potenciar las estrategias activas de afrontamiento como parte de las fortalezas personales con las que cuentan las personas con esquizofrenia. Aunque habitualmente las investigaciones han focalizado su atención en el estudio de las estrategias disfuncionales como la evitación o el aislamiento social (Vauth et al., 2007; Yanos et al., 2008), el presente estudio centra el interés en la beneficiosa asociación que podría establecerse entre el uso de estrategias adaptativas y activas de afrontamiento con mayores expectativas de recuperación. Junto a ello, la promoción de la autoestima y la implantación de estrategias que reduzcan la internalización del estigma podría contribuir a mejorar las expectativas sobre el propio proceso de recuperación. Finalmente el hecho que la recuperación subjetiva pueda ocurrir pese a la presencia de síntomas psiquiátricos (Andresen et al., 2010) sugiere que este criterio no debería ser empleado para excluir a las personas con mayor sintomatología o un peor funcionamiento psicosocial de los programas de promoción de la recuperación subjetiva.

\section{Referencias}

Andresen, R., Caputi, P. y Oades, L. (2006). Stages of recovery instrument: development of a measure of recovery from serious mental illness. Australian and New Zealand Journal of Psychiatry, 40(11-12), 972-980. 
Andresen, R., Caputi, P. y Oades, L. G. (2010). Do clinical outcome measures assess consumer-defined recovery? Psychiatry Research, 177(3), 309-317.

Andresen, R., Oades, L. y Caputi, P. (2003). The experience of recovery from schizophrenia: towards an empirically validated stage model. Australian and New Zealand Journal of Psychiatry, 37(5), 586-594.

Birchwood, M., Smith, J., Cochrane, R., Wetton, S. y Copestake, S. (1990). The Social Functioning Scale. The development and validation of a new scale of social adjustment for use in family intervention programmes with schizophrenic patients. The British Journal of Psychiatry, 157, 853-859.

Brohan, E., Elgie, R., Sartorius, N. y Thornicroft, G. (2010). Self-stigma, empowerment and perceived discrimination among people with schizophrenia in 14 European countries: the GAMIAN-Europe study. Schizophrenia Research, 122(13), 232-238.

Cano García, F. J., Rodríguez Franco, L. y García Martínez, J. (2007). Adaptación española del Inventario de Estrategias de Afrontamiento. Actas Españolas de. Psiquiatría., 35, 29-39.

Cavelti, M., Beck, E. M., Kvrgic, S., Kossowsky, J. y Vauth, R. (2012). The role of subjective illness beliefs and attitude toward recovery within the relationship of insight and depressive symptoms among people with schizophrenia spectrum disorders. Journal of Clinical Psychology, 68(4), 462-476.

Chiba, R., Miyamoto, Y. y Kawakami, N. (2010). Reliability and validity of the Japanese version of the Recovery Assessment Scale (RAS) for people with chronic mental illness: scale development. International Journal of Nursing Studies, 47(3), 314-322.

Connell, M., King, R. y Crowe, T. (2011). Can employment positively affect the recovery of people with psychiatric disabilities? Psychiatric Rehabilitation Journal, 35(1), 59-63.

Corrigan, P. W. (2006). Impact of consumer-operated services on empowerment and recovery of people with psychiatric disabilities. Psychiatric Services, 57(10), 1493-1496.

Corrigan, P. W., Giffort, D., Rashid, F., Leary, M. y Okeke, I. (1999). Recovery as a psychological construct. Community Mental Health Journal, 35(3), 231-239.

Corrigan, P. W. y Phelan, S. M. (2004). Social support and recovery in people with serious mental illnesses. Community Mental Health Journal, 40(6), 513-523.

Corrigan, P. W., Salzer, M., Ralph, R. O., Sangster, Y. y Keck, L. (2004). Examining the factor structure of the recovery assessment scale. Schizophrenia Bulletin, 30(4), 1035-1041.

Fernández, M. D., y Díaz, M. A. (2001). Relación entre estrategias de afrontamiento, síndromes clínicos y trastornos de personalidad en pacientes esquizofrénicos crónicos. Revista de Psicopatología y Psicología Clínica, 6(2), 129-135.

Fung, K. M., Tsang, H. W. y Corrigan, P. W. (2008). Self-stigma of people with schizophrenia as predictor of their adherence to psychosocial treatment. Psychiatric Rehabilitation Journal, 32(2), 95-104.

Gerlinger, G., Hauser, M., De Hert, M., Lacluyse, K., Wampers, M. y Correll, C. U. (2013). Personal stigma in schizophrenia spectrum disorders: a systematic review of prevalence rates, correlates, impact and interventions. World Psychiatry, 12(2), $155-164$
Hendryx, M., Green, C. A. y Perrin, N. A. (2009). Social support, activities, and recovery from serious mental illness: STARS study findings. The Journal of Behavioral Health Services \& Research, 36(3), 320-329.

Kay, S. R., Fiszbein, A. y Opler, L. A. (1987). The positive and negative syndrome scale (PANSS) for schizophrenia. Schizophrenia Bulletin, 13(2), 261-276.

Kukla, M., Lysaker, P. H. y Salyers, M. P. (2013). Do persons with schizophrenia who have better metacognitive capacity also have a stronger subjective experience of recovery? Psychiatry Reseach, 209(3), 381-385.

Lazarus, R. S. y Folkman, S. (1986). Estrés y procesos cognitivos. Barcelona: Martínez Roca.

Leamy, M., Bird, V., Le Boutillier, C., Williams, J. y Slade, M. (2011). Conceptual framework for personal recovery in mental health: systematic review and narrative synthesis. The British Journal of Psychiatry, 199, 445-452.

Liberman, R. P., Kopelowicz, A., Ventura, J. y Gutkind, D. (2002). Operational criteria and factors related to recovery from schizophrenia. International Review of Psychiatry, 14, 256-272.

Livingston, J. D. y Boyd, J. E. (2010). Correlates and consequences of internalized stigma for people living with mental illness: a systematic review and meta-analysis. Social Science \& Medicine, 71(12), 2150-2161.

Lloyd, C., King, R. y Moore, L. (2009). Subjective and objective indicators of recovery in severe mental illness: a cross-sectional study. International Journal of Social Psychiatry, 56(3), 220-229.

Lysaker, P. H., Roe, D. y Yanos, P. T. (2007). Toward understanding the insight paradox: internalized stigma moderates the association between insight and social functioning, hope, and self-esteem among people with schizophrenia spectrum disorders. Schizophrenia Bulletin, 33(1), 192-199.

McNaught, C., Caputi P., Oades, L. G. y Deane, F. P. (2007). Testing the validity of the Recovery Assessment Scale using an Australian sample. Australian and New Zealand Journal of Psychiatry, 41, 450-445.

Mukolo, A., Heflinger, C. y Baxter, J. (2011). Recovery and self-esteem: Concurrent validity of the Recovery Assessment Scale. International Journal Psychosocial Rehabilitation, 15(2), 41-68.

Muñoz, M., Sanz, M., Pérez-Santos, E. y Quiroga M. A. (2010). Proposal of a socio-cognitive-behavioral structural equation model of internalized stigma in people with severe and persistent mental illness. Psychiatry Research, 186(2-3), 402-408.

Peralta, V. y Cuesta, M. J. (1994). Psychometric properties of the positive and negative syndrome scale (PANSS) in schizophrenia. Psychiatry Research, 53(1), 31-40.

Ritsher, J. B., Otilingam, P. G. y Grajales, M. (2003). Internalized stigma of mental illness: psychometric properties of a new measure. Psychiatry Research, 121(1), 31-49.

Roe, D., Mashiach-Eizenberg, M. y Corrigan, P. (2012). Confirmatory factor analysis of the brief version of the recovery assessment scale. The Journal of Nervous and Mental Disease, 200(10), 847-851.

Roe, D., Mashiach-Eizenberg, M. y Lysaker, P. H. (2011). The relation between objective and subjective domains of recovery among persons with schizophrenia-related disorders. Schizophrenia Research, 131(1-3), 133-138. 
Rosenberg, M. (1965). Society and the adolescent self-image. Princeton, NJ: Princeton University Press.

Rüsch, N., Corrigan, P. W., Powell, K., Rajah, A., Olschewski, M., Wilkniss, S. y Batia, K. (2009). A stress-coping model of mental illness stigma: II. Emotional stress responses, coping behavior and outcome. Schizophrenia Research, 110(1-3), 65-71.

SAMHSA. (2005). National Consensus Conference on Mental Health Recovery and Systems Transformation, Rockville, MD.

Sibitz, I., Unger, A., Woppmann, A., Zidek, T. y Amering, M. (2009). Stigma resistance in patients with schizophrenia. Schizophrenia Bulletin, 37(2), 316-323.

Silverstein, S. M. y Bellack, A. S. (2008). A scientific agenda for the concept of recovery as it applies to schizophrenia. Clinical Psychology Review, 28(7), 1108-1124.

Teng, E. L. y Chui, H. C. (1987). The Modified Mini-Mental State (3MS) examination. Journal of Clinical Psychiatry, 48(8), 314-318

Tobin, D. L., Holroyd, K. A., Reynolds, R. V. y Kigal, J. K. (1989). The hierarchical factor structure of the Coping Strategies Inventory. Cognitive Therapy and Research, 13, 343-361.
Torres, A. y Olivares, J. M. (2005). Validation of the Spanish version of the Social Functioning Scale. Actas Españolas de Psiquiatría, 33.

Vauth, R., Kleim, B., Wirtz, M. y Corrigan, P. W. (2007). Self-efficacy and empowerment as outcomes of self-stigmatizing and coping in schizophrenia. Psychiatry Research, 150(1), 71-80.

Vázquez-Morejón, A. J., Jiménez García-Bóveda, R. y Vázquez-Morejón Jiménez, R. (2004). Escala de autoestima de Rosenberg: fiabilidad y validez en población clínica española. Apuntes de Psicología, 22, 247-255.

Yanos, P. T., Roe, D., Markus, K. y Lysaker, P. H. (2008). Pathways between internalized stigma and outcomes related to recovery in schizophrenia spectrum disorders. Psychiatric Services, 59(12), 1437-1442.

Young, S. L. y Bullock, W. A. (2003). The mental health recovery measure. In T. Campbell-Orde, J. Chamberlin, J. Carpenter, y H. S. Leff (Ed.), Measuring the Promise: A Compendium of Recovery Measures (Vol. II). Cambridge, MA. 